\title{
Primer congreso sobre la nutrición de los estratos pobres de la población: hacia una historia de la alimentación y nutrición en Chile (1931)
}

\author{
First congress on the nutrition \\ of the poorer strata of the population: \\ towards a history of feeding and \\ nutrition in Chile (1931)
}

Juan Carlos Yáñez A.

\begin{abstract}
This article studies the First Congress on the nutrition of the poorer strata of the population developed in the City of Valparaiso (Chile) in 1931. It is approached in the context of a conference, the role played by the first generation of doctors concerned about the problem of nutrition and the main conclusions of meeting. We note that the First Congress is the beginning of a nutrition policy to the poorer strata of the population that will be accompanied with the creation of the National Council of Food in 1937. Key words: congress; feeding; nutrition; poor; Chile.
\end{abstract}

Escuela de Administración Hotelera y Gastronómica, Universidad de Valparaíso.
Valparaíso, Chile.
Dirigir correspondencia a:
Juan Carlos Yáñez Andrade,
Escuela de Administración Hotelera y Gastronómica,
5 oriente No180, Viña del Mar,
Chile.
Correo electrónico: juancarlos.yanez@uv.cl

Este trabajo fue recibido el 18 de Noviembre de 2015 y aceptado para ser publicado el 10 de Junio de 2016.

\section{INTRODUCCIÓN}

Los primeros intentos por formular una política integral de alimentación datan de comienzos de los años 1930, producto de la crisis económica que afectaba al país. Por alimentación popular hacemos referencia a los debates sobre las formas de consumo y valor nutritivo de aquellos alimentos considerados fundamentales en la salud del pueblo. Las preocupaciones se remontan a fines del siglo XIX, centrándose especialmente en la producción y distribución de la leche, por su alto valor nutritivo, para las mujeres embarazadas, lactantes y niños (1). Sin embargo, la cesantía y el aumento del costo de la vida colocaron en el tapete público la necesidad de mejorar la alimentación del pueblo en su conjunto y no sólo de un grupo. Junto con las conocidas "ollas de pobres" se debatió sobre la educación alimentaria y el Ministerio del Trabajo difundió a partir de 1932 cartillas con la finalidad de orientar a la población sobre tipos de alimentos, su valor nutritivo y costos $(2,3)$. El objetivo central del presente artículo fue analizar la convocatoria al Primer Congreso de Alimentación Popular (PCAP) realizado en la ciudad de Valparaíso en 1931, así como sus principales debates y resoluciones. Nuestra hipótesis apunta a reconocer en este encuentro no sólo la primera instancia formal de análisis acerca de los desafíos de una política pública sobre alimentación, sino también la necesidad que tenía la comunidad médica de vincularse entre sí y presionar a las autoridades para que implementaran acciones concretas en ese ámbito (4-5).

Partimos de un diagnóstico crítico sobre los estudios que se han hecho a la evolución de la medicina en Chile, constatando la falta de investigaciones sistemáticas sobre la evolución de la alimentación y nutrición en el siglo $X X$. En algunos casos se abordan aspectos referidos a la cocina y las tradiciones culturales (6), pero muy poco los aspectos médicos, mientras que en otros se sitúan las políticas sobre la materia en los años 1940 (7-10).

En términos formales, estudiaremos el primer grupo de médicos interesados en la alimentación popular, especialmente en la ciudad de Valparaíso, para luego abordar las discusiones y conclusiones del PCAP. Por último, ofrecemos un anexo donde se presentan las resoluciones del congreso, 
las que por su interés histórico merecen ser conocidas en su integridad.

\section{MÉDICOS PRECURSORES}

El análisis del PCAP no puede estar ajeno al contexto histórico de la época, cruzado por la omnipresente "cuestión social", fenómeno caracterizado por el conjunto de problemas derivados de la industrialización y urbanización que Chile experimentó desde los años 1880 (11-13). Se multiplicaron en la prensa, en el Congreso Nacional y en las memorias universitarias, los debates y estudios sobre el alcoholismo, la prostitución, las viviendas insalubres y la mortalidad infantil, junto a otros flagelos que afectaban a los sectores populares (14-15). Las acciones de la beneficencia de raíz católica y de las sociedades de socorros mutuos -importantes durante gran parte del siglo XIX- se mostraron insuficientes para enfrentar una "cuestión social" que desbordaba los márgenes de la caridad y asociatividad (16-17).

La higiene fue el dispositivo central al cual apeló la comunidad médica en su lucha en contra de los problemas sociales, a lo que ayudó la naciente institucionalidad sanitaria del país. En 1892 se creó el Consejo Superior de Higiene Pública, que permitió centralizar las medidas sanitarias y consensuar políticas sobre la materia, mientras que en 1918 fue fundada la Dirección General de Sanidad, actuando como órgano ejecutivo y de fiscalización del recién promulgado Código Sanitario, a cargo del médico Ramón Corbalán Melgarejo (18).

Las primeras leyes sociales buscaron dar respuesta a los problemas sociales más urgentes, en particular la ley de habitaciones obreras (1906), la ley de descanso dominical (1907), la ley de sala cuna (1914) y la ley de accidentes del trabajo (1916) (19). Sin embargo, fue con la promulgación del paquete legislativo de septiembre de 1924 -fruto de un movimiento militar que derrocó al Presidente Arturo Alessandri- que se abordó de manera integral el problema social en Chile. En esa oportunidad se aprobaron ocho leyes sociales, destacando la ley N4054, impulsada por el médico Exequiel González Cortés, que instauró la seguridad social (20).

Por último, un paso importante en el diseño de políticas sanitarias lo constituyó la creación en 1924 del Ministerio de Higiene, Asistencia, Previsión Social y Trabajo, cuyo primer ministro fue el médico Alejandro del Rio.

A nivel profesional, los médicos desde muy temprano buscaron organizarse gremialmente, fundando la Sociedad Médica de Santiago en 1869, de Concepción en 1893 y de Valparaíso en 1913. Esta última jugó un papel fundamental en la defensa de los intereses del sector médico del puerto y en el fortalecimiento de la asistencia pública y sanitaria de la ciudad, teniendo una destacada participación los médicos Federico Engelbach, Plutarco Badilla, José Grossi, Hugo Grove y Gustavo Fricke, entre otros (21). En 1924, gracias a la nueva ley de sindicalización profesional, se fundó en Valparaíso el Sindicato de Médicos de Chile con la participación de algunos miembros de la Sociedad Médica de esa ciudad, con la finalidad explícita de luchar por "el mejoramiento moral y económico de la profesión" y por la correcta aplicación de la ley de seguro social (22). En términos políticos, si bien no tuvo una posición partidaria definida, el Sindicato se manifestó de acuerdo con el proceso de transformaciones sociales que el país vivía en la época y cuestionó a la Sociedad Médica de Santiago por sus enfoques tradicionales y su excesivo centralismo (23).

La Sociedad Médica de Valparaíso fue la responsable de promover en las páginas de su revista -Revista de la Sociedad Médica de Valparaíso, entre 1926 y 1929- estudios sobre la nutrición, la mortalidad infantil y los beneficios de la lactancia (24). En 1930 la Sociedad comenzó a publicar la Revista de la Alimentación Popular, bajo la dirección del doctor Germán Vogel, para que sirviera de órgano de propaganda en la realización del PCAP. Si bien tuvo sólo dos números y terminó su circulación una vez finalizado el encuentro, sirvió como espacio de debate y de difusión de las actividades de una primera generación de médicos preocupados por la alimentación de la población de escasos recursos. Además de publicar estudios sobre nutrición, la revista fue importante en la recaudación de recursos para el congreso, a través de la adhesión y pago de las respectivas cuotas. Se informó de la participación de numerosas municipalidades, instituciones científicas y organizaciones profesionales, pero ante la falta de dinero se solicitó ayuda al Estado, para lo cual se contó con el apoyo del diputado José Manuel Ríos (25).

El Comité Ejecutivo del PCAP comenzó a reunirse de manera permanente desde comienzos de 1930 en los locales de la Sociedad Médica de Valparaíso y bajo la presidencia de Vicente Dagnino, médico de dilatada trayectoria en el campo del alcoholismo y epidemiología (26). Lo secundaban los médicos Hans Bethzold, Edwyn Reed, Carlos Schwarzenberg, Enrique Valenzuela Rosas, Humberto Vera y Germán Vogel como secretario y Ernesto Ewertz como tesorero. Una de las funciones principales del Comité Ejecutivo era la coordinación de los distintos expositores que se presentarían al PCAP, a fin de dar cumplimiento al programa propuesto, el cual estaba dividido en cinco secciones: médica; bromatología e inspección sanitaria; producción, abastecimiento y comercio; legislación y economía social; educación y propaganda (27).

\section{PRIMER CONGRESO DE ALIMENTACIÓN POPULAR}

El 24 de enero de 1931 se inauguró en la ciudad de Valparaíso el PCAP, bajo la iniciativa de la Sociedad Médica de esa ciudad. Si bien sus alcances específicos son difíciles de medir, estamos en condiciones de señalar que expresó el deseo de un grupo de médicos de conducir el debate público y científico con la finalidad de formular políticas públicas sobre alimentación. Esto aparece evidente al constatar la seriedad con que los profesionales asumieron el desafío de presentar sus trabajos y exponerlos al debate crítico de la comunidad científica, así como por su diversidad y número, los que alcanzaron el medio centenar. Presidente del Congreso fue electo por unanimidad el destacado médico Luis Calvo Mackenna y como Secretario General Germán Vogel.

De las cinco secciones originales en las que estaba organizado el encuentro se lograron reunir solo tres: médica, bromatología y economía social. Entre las presentaciones destacan temas tan variados como el problema del raquitismo, los regímenes alimentarios en guarniciones y cárceles, diversos estudios sobre el pan, las vitaminas, la calidad de las harinas, métodos analíticos de la bromatología, microbiología de los alimentos, desayuno escolar, ferias libres y la enseñanza de la alimentación en los colegios, entre otros (28). Dentro de las actividades complementarias al congreso, estuvo la visita realizada a algunas fábricas de Valparaíso con el fin de conocer las técnicas de producción y la realización de un muestrario de alimentación, donde se exhibieron alimentos indicando su valor nutritivo y costo.

Entre las conclusiones del PCAP se encuentran el mejoramiento agrícola, el uso de abonos y la promoción del consumo de pan integral y de azúcares naturales, como la miel y la uva. En el aspecto institucional se propuso la creación de un 
organismo coordinador de políticas alimentarias, denominado Consejo Nacional de Subsistencia y Nutrición, que colaborara con el Comité de Higiene de la Sociedad de Naciones en la realización de encuestas. A nivel de gestión local se solicitó que los municipios mejoraran la administración de los mataderos, promovieran el uso de ferias libres y de restaurantes económicos, junto a la creación de laboratorios de control de alimentos. Por último, en cuanto a educación, se propuso la incorporación de la enseñanza agrícola y doméstica en las escuelas públicas (29).

\section{CONSIDERACIONES FINALES}

Los efectos de la crisis económica que afectaba a Chile, y a gran parte del mundo, producto de la Gran Depresión, fueron importantes para movilizar el interés y las energías de una primera generación de médicos preocupados por el problema de la alimentación de las clases trabajadoras y convocar al PCAP. Sin embargo, médicos como Luis Calvo Mackenna, Gustavo Fricke, José Grossi, Vicente Dagnino y Hugo Grove, por citar algunos, venían jugando, desde fines del siglo XIX, un papel fundamental en proponer las primeras medidas de cuidado a la infancia, de mejoramiento de la producción y consumo de leche, de perfeccionamiento de una institucionalidad sanitaria, entre muchas otras. Muchas de las conclusiones del PCAP tuvieron la posibilidad de ver la luz en realizaciones concretas y nutrir el debate de los años 1930 y 1940, bajo la conducción de una nueva generación de médicos: Eduardo Cruz-Coke, Jorge Mardones Restat y Julio Santa María, entre otros.

El Ilamado a colaborar con organismos internacionales respondió al hecho de que los debates sobre alimentación y nutrición se habían transformado en objeto de política internacional en el seno de las organizaciones supra gubernamentales que formaban parte de la Sociedad de Naciones (30). Por ejemplo, la Organización Internacional del Trabajo fue receptiva al tema de la nutrición, porque tenía relación con el costo de la vida y la salud de los trabajadores, todo enmarcado en la crisis económica de los años 1930 que hacía difícil que la población alcanzara estándares mínimos de vida (31). Entonces no es extraño que en 1933 el gobierno chileno solicitara una comisión de encuesta internacional para estudiar el problema de la alimentación en el país. La investigación quedó a cargo de los doctores Carlos Dragoni y Etienne Burnet, funcionarios de la SDN, siendo realizada en el transcurso de 1935. El estudio demostró las serias deficiencias en alimentación que la población chilena arrastraba por décadas. Sólo 30\% de las familias encuestadas consumían más de 3.000 calorías diarias recomendables en la época (32).

Este primer periodo finaliza, en materia institucional, con la creación en 1937 del Consejo Nacional de Alimentación, bajo el apoyo del Ministro de Salud Eduardo Cruz-Coke, y cuya dirección estuvo a cargo de Jorge Mardones Restat, lo que significó la consagración de la alimentación como objeto de política pública.

\section{ANEXO \\ CONCLUSIONES PRIMER CONGRESO DE ALIMENTACIÓN POPULAR}

$1^{\circ}$ Recomendar la sindicalización obligatoria de las industrias extractivas, agrícolas y manufactureras.

$2^{\circ}$ La creación de un Consejo Nacional de Subsistencias y Nutrición.

$3^{\circ}$ Que se hagan efectivas las franquicias que las leyes vigentes acuerdan a las diversas clases de cooperativas, por ser éstas la mejor organización y defensa contra el encarecimiento de la vida.

$4^{\circ}$ Que las ayudas y subvenciones especiales que el Gobierno otorga a las Cooperativas de Producción, se mantengan y prosperen con la debida continuidad para asegurar su desarrollo.

$5^{\circ} \quad$ La revisión de los Estatutos y el fin social de toda institución que lleve el nombre de cooperativa, prohibiendo funcionar a las que no cumplan con su objeto, y no autorizar nuevas Cooperativas sin el informe favorable del futuro Consejo Nacional de Subsistencias y de Nutrición o de la actual Oficina de Cooperativas.

$6^{\circ}$ Las ferias francas, siempre que funcionen en sitios especialmente arreglados para este objeto, que reúnan condiciones sanitarias adecuadas y sean sometidos a reglamentación profiláctica los artículos en venta y el personal encargado de ella.

$7^{\circ}$ La formación del mayor número posible de Restaurantes Económicos Cooperativos.

$8^{\circ}$ El incremento de la enseñanza pública de la chacarería y fruticultura, así como apicultura en las escuelas rurales, adquiriendo granjas o predios adecuados, dándose mayor desarrollo a las cantinas escolares, a las cuales se destinarán los productos obtenidos.

$9^{\circ}$ Estimular en la enseñanza escolar, la difusión del sistema cooperativo, como el mejor medio de satisfacer las necesidades sociales.

$10^{\circ}$ Dar mayor importancia a la enseñanza de economía doméstica en los liceos y escuelas, adoptando un texto especial y un programa general para todos los establecimientos, con lecciones teóricas y prácticas.

$11^{\circ}$ Adherirse al plan de Fomento Pesquero, que está desarrollando el Supremo Gobierno; reconocer que la organización en cooperativas, de los gremios de pescadores, es el mejor medio de eliminar intermediarios que encarecen el precio del pescado, así como la distribución de este artículo en frigoríficos y mercados públicos, recomendando a todos sus miembros la más efectiva propaganda en favor del consumo de pescado, como uno de los más positivos medios para mejorar las condiciones de vida, contribuir a su abaratamiento y hacer de esta industria una fuente de grandes riquezas exportables.

$12^{\circ}$ Establecer el sobresalario familiar, ya implantado en casi todos los países de racional y científica estructura social.

$13^{\circ}$ El mantenimiento de la ley que prohíbe el trabajo nocturno en las panaderías, y pedir al Gobierno que sus Legaciones y Consulados informen sobre los progresos alcanzados en la mecánica de la industria del pan, con presupuesto, maquinaria, forma de explotación, rendimiento, entre otros, a fin de reglar en la forma más conveniente el trabajo en dichos establecimientos.

$14^{\circ}$ Solicitar de los Ferrocarriles del Estado que, para el transporte de la harina, se empleen carros que no pongan en peligro su calidad.

$15^{\circ}$ Declarar constituida la "Asociación de Alimentación Nacional" sobre la base de los miembros del actual Congreso, con el fin de continuar los estudios de la alimentación, su forma económica y difusión, contando como órgano de publicidad con la "Revista de la Alimentación Popular", y facultar a la mesa directiva para redactar las bases y los estatutos correspondientes.

\section{RESUMEN}

En este artículo se estudia el Primer Congreso sobre la nutrición de los estratos más pobres de la población, desarro- 
Ilado en la ciudad de Valparaíso (Chile) en 1931. Se aborda en el contexto de la conferencia, el papel jugado por la primera generación de médicos preocupados por el problema de la nutrición y las principales conclusiones del encuentro. Señalamos que el Primer Congreso es el inicio de una política de nutrición de los estratos más pobres de la población, lo que será acompañado con la creación del Consejo Nacional de Alimentación en 1937. Chile.

Palabras clave: congreso; alimentación; nutrición; pobres;

Agradecimientos: El presente artículo forma parte del Proyecto Fondecyt de Investigación Postdoctoral N³160471 titulado "Inequidad alimentaria y estándar de vida. Las políticas de alimentación popular en el Chile de entre guerra: 1920-1950".

\section{BIBLIOGRAFÍA}

1. Zárate, M. S. "The liquor of life. Breastfeeding and maternal and infant feeding, Chile (1900-1950)" in Sciolla, C. (Editor), History and culture of food in Chile. Catalonia, Santiago, 2013.

2. Salas, D. Newsletter of food. Ediciones Restaurant Naturista, Santiago, 1932.

3. Gómez, J. C. "Crisis, hunger and socialism in Chile, 19311932", Andes, 1988 (7): 101- 59.

4. Vargas, J. E. "Features of social and professional selfimage of doctors (1872- 1925)", Ars Médica, 2001; 3 (4): $103-26$.

5. Larraín, C. The Medical Society of Santiago and the historical development of medicine in Chile. Sociedad Médica de Santiago, Santiago, 2002.

6. Plath, O. Food and folklore language (no editorial data), Santiago, 1949.

7. Cruz-Coke, R. History of Chilean medicine. Edit. Andrés Bello, Santiago, 1995.

8. Crovetto, M. "Evolution of nutrition and food in Chile in the twentieth century", Rev Chil Nutr. 2002; 29 (3): 316-7.

9. Mardones-Restat, F. "Food and nutrition policies in the health plans", Rev Chil Nutr. 2003; 30 (suppl. 1): 195-7.

10. Valiente, S. y Uauy, R. "Development of nutrition and food in Chile" in Bourgues, H., Bengoa J. and O'Donnell, A. History of Nutrition in Latin America (no editorial data), http://www.sochinut.cl/pdf/HistNut_SLAN.Pdf (consulta 15 marzo 2015)

11. Morris, J. Elites, intellectuals, and consensus; a study of the social question and the industrial relations system in Chile. Editorial del Pacífico, Santiago, 1967.
12. Grez, S. The "social question" in Chile. Precursor ideas and discussions (1804-1902). Dibam, Santiago, 1995.

13. Yáñez, J. C. State, consensus and social crisis. The public space in Chile: 1900-1920. Dibam, Santiago, 2003.

14. Fernandéz, M. Alcoholic beverages in Chile: an economic history of their development and expansion, 1870-1930. Ediciones Universidad Alberto Hurtado, Santiago, 2010.

15. Yáñez, J.C.(Editor). Alcohol and Work: The Alcohol and the making of labor identities. Chile, nineteenth and twentieth century. Editorial Universidad de Los Lagos, Osorno, 2008.

16. Ponce de León, M. Governing poverty. Charity practices and welfare in the city of Santiago, 1830-1890. Dibam, Santiago, 2011.

17. Illanes, M. A. Solidarity revolution. History of the mutual aid societies workers. Chile, 1840-1920. Colectivo de Atención Primaria, Santiago, 1990.

18. Illanes, M. A., In the name of the People, the State and the Science. Colectivo de Atención Primaria, Santiago, 1993.

19. Yáñez, J. C. "Background and historical development of social legislation in Chile between 1906 and 1924". Rev Est Hist Jurid. 1999 (21): 203-10.

20. Yáñez, J. C. Social intervention in Chile (1907-1932). Ril Editores, Santiago, 2008.

21. Cruz-Coke, R. History of Chilean medicine. Editorial Andrés Bello, Santiago, 1995.

22. Medical Union of Chile, Valparaíso, 1925 (1): 1-2.

23. Molina, C. Chilean health institutions, 1889-1989. Lom Ediciones, Santiago, 2010.

24. The Journal of the Medical Society of Valparaiso, directed by Federico Engelbach.

25. J Nutr. poor. 1930 (2): 27-32.

26. Dagnino, V. "Causes of dissolution of the family and child mortality". Rev Soc Med Valp. 1928 (10): 693-703.

27. J Nutr. poor. 1930 (1): 32.

28. Background and works of the First National Congress of nutrition of the poor. Imprenta Aurora, Santiago, 1932.

29. Martínez, M. Modalities to reduce food and conclusions of the First National Congress of nutrition of the poor. Imprenta Casa Amarilla, Santiago, 1931, 18-29.

30. Pernet, C. "The ILO and the question of food supply in Latin America (1930-1950)", in Lespinet-Moret, I. and Viet, V. The International Labour Organization. Presses Universitaires de Rennes, Rennes, 2011.

31. Barona, J. "Nutrition and Health. The International Context during the Inter-War Crisis", Soc Hist Med. 2008; 21(1): 87-105.

32. Dragoni, C. and Burnet, E. Nutrition of the poor: first general survey 1935. Imprenta Universo, Santiago, 1938. 